\title{
Tendencias de los Artículos en Psicología Clínica en Iberoamérica
}

\author{
Tendencies in Clinical Psychology Articles in Iberoamerica
}

\author{
César Armando Rey Anacona* \\ Jorge Arturo Martínez Gómez \\ Smith Ibeth Guerrero Rodríguez \\ Universidad Pedagógica y Tecnológica de Colombia \\ (Rec: 06 mayo 2009 Acep: 07 junio 2009)
}

\begin{abstract}
Resumen
Se analizaron las tendencias de los artículos científicos en psicología clínica en Iberoamerica con base en el número de artículos que se pueden recuperar a través de los sistemas de información REDALYC y PSICODOC, en las áreas de psicopatología, evaluación psicológica, diagnóstico psicológico, formación del psicólogo, enfoque terapéutico y psicoterapia, publicados entre los años 2003 y 2007. Para las áreas "Psicopatología" y "Enfoque terapéutico" se delinearon además otras palabras claves que permitieran realizar un análisis más comprehensivo de sus tendencias. La búsqueda se realizó en el campo "Resumen" (en el área de conocimiento "Psicología") y "Búsqueda asistida", respectivamente. El indicador muestra un visible interés por la psicopatología, la psicoterapia y la evaluación psicológica y uno más bajo con respecto a la formación del psicólogo y el diagnóstico psicológico. La ansiedad se observa como el tema de mayor interés con respecto a la psicopatología, mientras que la terapia cognitiva y la terapia conductual lo fueron en relación con el enfoque terapéutico. Se plantean otras tendencias con base en el mismo indicador.
\end{abstract}

Palabras claves: Psicología clínica, investigación científica, programas de investigación.

\begin{abstract}
Tendencies of clinical psychology scientific articles were analyzed in Iberoamerica, based upon the number of articles that could be recovered by the systems REDALYC and PSICODOC in the areas of Psychopathology, Psychological Evaluation, Psychological Diagnostic, Psychologist's Formation, Therapeutic Model and Psychotherapy, all published among 2003-2007. For the areas of Psychopathology and Therapeutic model were delineated additionally other key words for a more comprehensive analysis. The search was carried out in the field "Summarize" ("Psychology" as knowledge area) and "Advanced search", respectively. The indicator shows a visible interest for the topics Psychopathology, Psychotherapy and Psychological Evaluation and lower internet with regard to Psychologist's Formation and Psychological Evaluation. Anxiety is observed as the topic of more interest in psychopathology, while cognitive and behavioral therapy were notable in psychotherapy. Other tendencies were outlined based in the same indicator.
\end{abstract}

Key words: Clinical psychology, scientific investigation, research programs.

* Correspondencia: Calle 24 No. 5-63, Antiguo Hospital San Rafael, Escuela de Psicología, Facultad de Ciencias de la Salud, Universidad Pedagógica y Tecnológica de Colombia, Tunja, Colombia. Correo electrónico: cesar.rey@uptc.edu.co. 
La psicología clínica es una de las áreas profesionales más dinámicas de la psicología por sus diferentes ámbitos de desempeño, que la han llevado a relacionarse con disciplinas y profesiones tales como la psiquiatría, la reeducación, el derecho, la neurociencia y la psicopatología. Si bien para autores como Peña (2005), los psicólogos clínicos se dedican al diagnóstico y el tratamiento psicológico, otros como Resnick (1991) resaltan que éstos además de estas actividades puramente profesionales, se dedican a otras como la consultoría, la enseñanza y la investigación. Siguiendo a Trull y Phares (2003), se puede plantear que los psicólogos en el campo clínico se dedican a las siguientes actividades:

1. El diagnóstico y la evaluación psicológica, por medio de las cuales observan, cuantifican y caracterizan las capacidades, los problemas y las limitaciones psicológicas y sociales de los consultantes.

2. La intervención, mediante la planificación y ejecución de programas de terapia individual o grupal, derivados de principios teóricos propios de la psicología, donde el terapeuta y el consultante establecen objetivos tendientes a mejorar la calidad de vida de este último.

3. La consulta, ofreciendo asesoría a diferentes tipos de organizaciones.

4. La enseñanza y la investigación, desempeñándose en universidades a través de cursos sobre técnicas de entrevista, pruebas psicológicas, personalidad, psicoterapia o de introducción a la psicología clínica, realizando actividades de supervisión de las prácticas de estudiantes de pregrado o postgrado y participando en proyectos de investigación en algunos de los campos teóricos o aplicados de la disciplina.

5. La administración, ocupando cargos directivos en universidades, en instituciones sanitarias o gubernamentales o participando en comités administrativos.

Además de estos múltiples roles, la psicología clínica presenta diferentes orientaciones que evidencian distintas visiones sobre el comportamiento desadaptativo o anormal y su evaluación y tratamiento. Dentro de éstos se pueden resaltar la perspectiva psicodinámica, el enfoque humanista, la orientación sistémica y la terapia cognitivo conductual (Nietzel, Bernstein \& Milich, 1998). Ante esta diversidad de enfoques que da lugar a diferentes técnicas de intervención, en los últimos años se ha resaltado la necesidad de sustentar empíricamente los tratamientos, con el fin de que los usuarios reciban una atención de mejor calidad, mejore la confianza del público en la psicoterapia y se incremente el posicionamiento de la disciplina y la profesión psicológicas (Londoño \& Valencia, 2005). Este movimiento de soporte empírico de las psicoterapias surgió a raíz de un informe que un equipo de trabajo liderado por D. L. Chambless presentó a la División de Psicología Clínica de la Asociación Psicológica Americana, en el cual se delimita una serie de criterios para definir los tres tipos de terapias psicológicas con soporte empírico: Las terapias "Bien establecidas", las "Probablemente eficaces" y "En fase experimental" (Chambless \& Holon, 1998).

Una de las consecuencias de este informe fue un incremento en la importancia de la "práctica clínica basada en la evidencia" (Llobell, Frías \& Monterde, 2003, p. 1), como una forma de facilitar el acercamiento entre la práctica profesional y la investigación científica y asegurar la calidad de los servicios del psicólogo clínico, importancia que debería reflejarse en un aumento en el número de artículos y otras publicaciones científicas sobre temas como la psicoterapia, la psicopatología, la evaluación psicológica, el diagnóstico psicológico, la formación del psicólogo y los enfoques terapéuticos. Un análisis de las tendencias de este tipo de publicaciones permite delinear la capacidad de producción científica de un país o una región en relación con estos temas y establecer necesidades a futuro sobre los esfuerzos investigativos que podrían ocurrir en dicho país o región (Vera-Villarroel \& Lillo, 2006).

En Iberoamérica se han publicado dos análisis de las tendencias de los artículos científicos sobre psicología clínica, incluyendo un análisis bibliométrico de los artículos de revistas de psicología clínica editadas en castellano que se publicaron entre los años 2001 y 2002 (Agudelo, Bretón-López \& Buela-Casal, 2003), en el cual se encontró que los principales campos de conocimiento de estos artículos estaban relacionados con la psicología clínica ("Personalidad, evaluación y tratamiento psicológico", "Ínter-área", "Metodología” y "Salud"), aunque había otras menos conectadas con el mismo ("Social", "Básica" y "Evolución y educación"). El otro análisis se centró en los artículos en psicología clínica de autores chilenos entre los años 2000 a 2006 (Vera-Villarroel \& Lillo, 2006), en el cual se examinó cuantitativamente la producción por autor y por universidad con base en el número de artículos publicados en revistas incluidas en los sistemas de información PSYCINFO e ISI WEB OF KNOWLEDGE. No obstante, ninguno de estos estudios exploró las tendencias de los artículos científicos en Iberoamerica con base en las principales áreas de la psicología clínica, ni tampoco incluyeron todas las revistas de psicología y no solamente las revistas de psicología clínica o aquellas indexadas por PSYCINFO o ISI WEB OF KNOWLEDGE. Por lo tanto, sus resultados son limitados para plantear tendencias en la región sobre los intereses académicos actuales y futuros en esta área de la psicología.

Con base en lo anterior, el objetivo de este trabajo fue realizar un estudio exploratorio de carácter bibliométrico sobre las tendencias de dichos artículos, basado en el número de este tipo de publicaciones que se pueden recuperar de los sistemas de información REDALYC y PSICODOC, a partir de un conjunto de palabras claves referentes a las principales áreas de la psicología clínica. Estas son: Psicopatología, evaluación psicológica, diagnóstico psicológico, formación 
del psicólogo, enfoque terapéutico y psicoterapia, realizándose adicionalmente un análisis más específico a nivel de los campos psicopatología y enfoque terapéutico.

\section{Método}

\section{Unidades de análisis}

Fueron los artículos publicados entre 2003 y 2007, cuyo texto completo o resumen se podía recuperar a través de los sistemas de información REDALYC y PSICODOC, por medio de una búsqueda realizada en el campo "Resumen" y "Búsqueda asistida", respectivamente. En el caso específico de REDALYC, esta búsqueda se llevó a cabo seleccionando "Psicología" como área de conocimiento de las revistas.

\section{Materiales}

REDALYC es la Red de Revistas Científicas de América Latina y el Caribe, España y Portugal, auspiciada por la Universidad Autónoma del Estado de México, con el objetivo de contribuir a la difusión de la actividad científica editorial que se produce en Iberoamérica (REDALYC, 2008, noviembre 17). Permite recuperar en texto completo los artículos de 549 revistas científicas, 40 de las cuales tienen como área principal o secundaria la psicología. PSICODOC, por su parte, es una base de datos bibliográfica producida por el Colegio Oficial de Psicólogos de Madrid, con enlaces a los textos completos y resúmenes de artículos de 617 revistas de psicología y disciplinas afines e incluye los trabajos publicados en revistas, congresos y libros, editados en España y América Latina (PSICODOC, 2008, noviembre 17).

Se seleccionó a REDALYC debido a su creciente difusión en la región, ya que permite recuperar libremente artículos en texto completo y a PSICODOC porque incluye un número mayor de revistas en psicología y disciplinas afines que el sistema de información LATINDEX, el tercer principal sistema en la región, que incluye la información de 584 revistas de psicología (LATINDEX, 2008, noviembre 17).

\section{Procedimiento}

Las palabras claves utilizadas en la búsqueda con cada sistema de información fueron: a) Psicopatología, b) evaluación psicológica, c) diagnóstico psicológico, d) formación del psicólogo, e) enfoque terapéutico y f) psicoterapia, registrándose el número de artículos que se encontraba con cada sistema y el número total de artículos encontrados con los dos. Para el caso particular de los campos "Psicopatología" y "Enfoque terapéutico", se delineó además una serie de palabras claves que permitieran realizar un análisis más comprehensivo de sus tendencias.
Para psicopatología se utilizaron las siguientes palabras claves: a) Ansiedad, b) estado de ánimo, c) psicótico, d) trastornos alimentarios, e) trastornos de personalidad, f) retraso mental, g) trastorno cognoscitivo, h) trastornos disociativos, i) trastornos sexuales, j) trastorno del sueño, k) somatomorfos, l) control de impulsos, m) trastornos de conducta, n) trastornos infantiles, o) depresión y p) trastorno bipolar. Estas palabras responden a las áreas generales delineadas por la Asociación Psiquiátrica Americana (APA) en la última versión de su manual diagnóstico (APA, 2002) y son los que frecuentemente se encuentran en los libros de texto sobre conducta anormal y psicopatología (e. g., Barlow \& Durand, 2001; Halgin \& Whitbourne, 2004; Sarason \& Sarason, 1996; Sue, Sue \& Sue, 1996).

Por su parte, para el campo de enfoque terapéutico se emplearon las siguientes palabras claves: a) Terapia cognitiva, b) terapia de conducta, c) terapia cognitiva conductual, d) terapia sistémica, e) terapia humanista y f) psicodinámica, las cuales obedecen a los enfoques terapéuticos prevalentes en la actualidad, como se desprende de los libros de texto sobre el tema (e. g., Nietzel, Bernstein \& Milich, 1998; Trull \& Phares, 2003). La búsqueda se realizó entre octubre y noviembre de 2008 y abarcó los últimos cinco años (20032007), registrándose el número de artículos por año y por país de origen de las revistas.

\section{Resultados}

\section{Psicopatología}

Con la palabra clave "Psicopatología" se encontró un total de 769 artículos en los dos sistemas de información, siendo el año 2004 el de mayor producción con 190 artículos (véase la Tabla 1 y la Figura 1). A continuación se presentan los resultados obtenidos con las palabras claves específicas del campo (véase la Tabla 2 y la Figura 2).

Ansiedad. En relación con la palabra clave "Ansiedad", en la búsqueda realizada en el sistema de información de REDALYC se encontraron 180 artículos de revistas que contenían la palabra clave "Ansiedad" en su resumen, de los cuales 95 eran de revistas españolas; luego se encuentra Colombia con 31 artículos y le siguen México y Brasil, cada uno con 14 artículos. Por su parte, en PSICODOC se hallaron 485 artículos de revistas que contenían la palabra "Ansiedad" en su resumen. Los países con mayor producción fueron España con 362 artículos de revista, Colombia con 30 artículos, México con 23 artículos y Argentina también con 23 artículos. En total se encontraron 665 artículos de revistas y el país que reportó en la búsqueda el mayor número de coincidencias fue España, con 457 artículos de revista. Lo sigue Colombia con 61 artículos, luego México con 37 artículos, Brasil con 32 artículos y Argentina con 27 artículos. 
Tabla 1. Número total de artículos publicados en Iberoamérica entre los años 2003 a 2007, en las principales áreas de la psicología clínica.

\begin{tabular}{lcccccc}
\hline Área & 2003 & 2004 & 2005 & 2006 & 2007 & Total \\
\hline Psicopatología & 155 & 190 & 164 & 166 & 94 & 769 \\
Enfoque terapéutico & 22 & 25 & 35 & 55 & 17 & 154 \\
Formación del psicólogo & 2 & 5 & 5 & 2 & 1 & 15 \\
Diagnóstico psicológico & 1 & 4 & 3 & 2 & 2 & 12 \\
Evaluación psicológica & 190 & 173 & 151 & 173 & 86 & 773 \\
Psicoterapia & 217 & 272 & 211 & 156 & 119 & 975 \\
Total & 587 & 669 & 569 & 554 & 319 & 2698 \\
\hline
\end{tabular}

Nota. Fuente: REDALYC y PSICODOC.

Figura 1. Número total de artículos publicados en Iberoamérica entre los años 2003 a 2007, en las principales áreas de la psicología clínica.

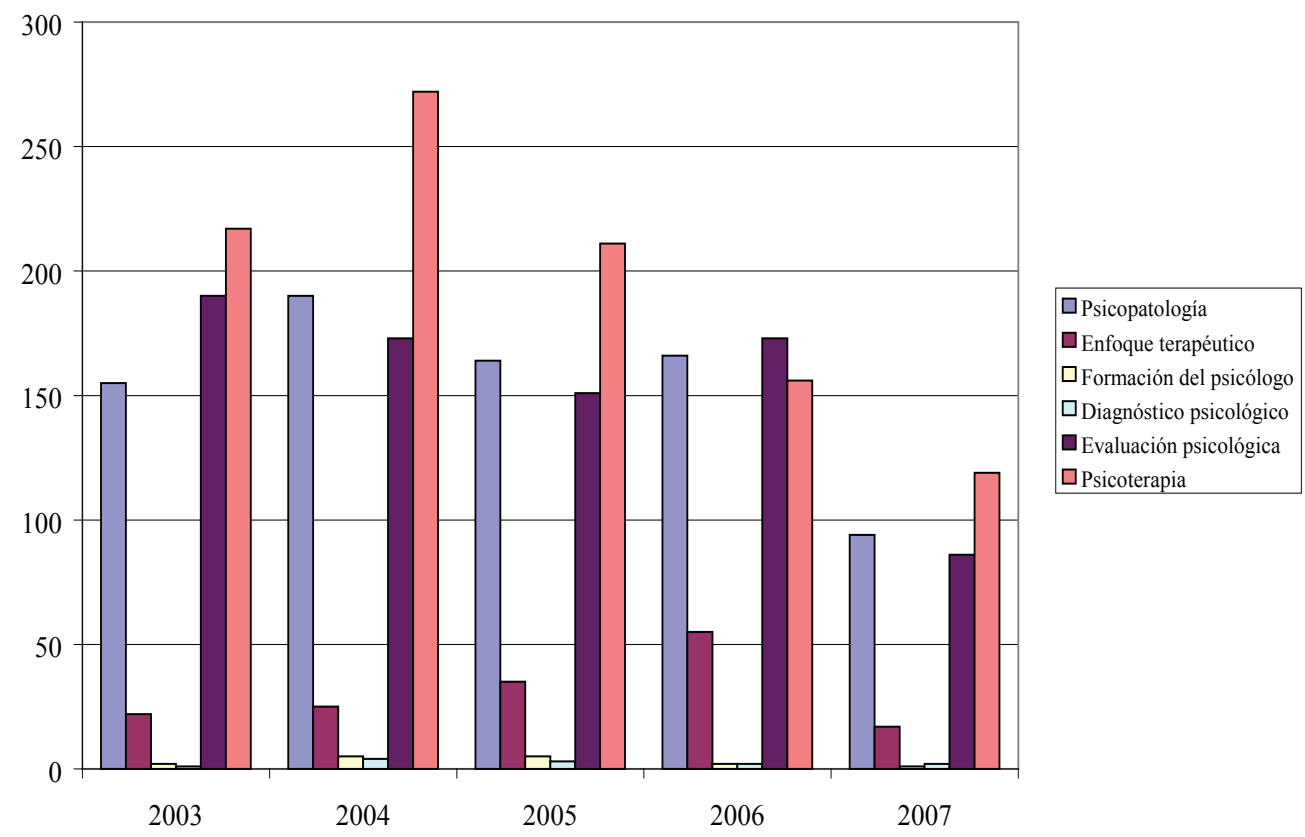

Nota. Fuente: REDALYC y PSICODOC.

Estado de ánimo. Con "Estado de ánimo" como palabras claves, se encontraron 10 artículos en el sistema de información de REDALYC. Los países que mostraron una mayor producción en el área fueron España con siete artículos y Colombia con tres. Con las mismas palabras, la base datos de PSICODOC arrojó un total de 28 artículos, 16 de revistas españolas, cuatro de revistas argentinas, tres de revistas brasileras, tres de revistas colombianas y dos de revistas chilenas. El número de artículos encontrados en el sistema de información de REDALYC y PSICODOC, con la palabra "Estado de ánimo", fue 38 en total.

Psicótico. Con la palabra "Psicótico" se encontró 46 artículos en la búsqueda en REDALYC, 19 de los cuales eran de revistas brasileñas y 12 de revistas españolas. La misma palabra en PSICODOC arrojó como resultado 72 artículos de revistas, de los cuales 43 eran de revistas españolas, ocho de Colombia, seis de Brasil y seis de México. En total se encontraron 118 artículos, en donde el año con mayor producción fue 2005 con 37 artículos. Brasil y España fueron los países con mayor número de artículos publicados.

Trastornos alimentarios. Utilizando estas palabras claves en la búsqueda de REDALYC, se encontró cinco artículos, de los cuales tres eran de revistas de España, uno de México y uno de Argentina. Con las mismas palabras en PSICODOC se halló 39 artículos, de los cuales se reportaron 24 artículos de España, seis de Colombia, cinco de Brasil, tres de México y uno de Venezuela. En total se encontraron 44 artículos de revistas en la búsqueda 
Tabla 2. Número total de artículos publicados en Iberoamérica entre los años 2003 a 2007, en áreas del campo psicopatología.

\begin{tabular}{|c|c|c|c|c|c|c|}
\hline Área & 2003 & 2004 & 2005 & 2006 & 2007 & Total \\
\hline Ansiedad & 148 & 142 & 144 & 133 & 98 & 665 \\
\hline Estado de ánimo & 7 & 12 & 4 & 9 & 6 & 38 \\
\hline Psicótico & 21 & 16 & 37 & 20 & 24 & 118 \\
\hline Trastornos alimentarios & 10 & 17 & 4 & 10 & 3 & 44 \\
\hline Trastornos de personalidad & 68 & 100 & 72 & 50 & 37 & 327 \\
\hline Retraso mental & 76 & 52 & 49 & 68 & 62 & 307 \\
\hline Trastorno cognoscitivo & 14 & 16 & 19 & 25 & 19 & 93 \\
\hline Trastornos disociativos & 2 & 3 & - & 1 & - & 6 \\
\hline Trastornos sexuales & 13 & 12 & 7 & 12 & 5 & 49 \\
\hline Trastorno del sueño & 35 & 14 & 16 & 12 & 8 & 85 \\
\hline Control de impulsos & 3 & 2 & 8 & 8 & 12 & 33 \\
\hline Somatomorfos & 2 & 1 & 2 & - & 1 & 6 \\
\hline Trastorno de conducta & 116 & 115 & 87 & 86 & 52 & 456 \\
\hline Trastornos infantiles & 1 & 2 & 3 & 3 & 2 & 11 \\
\hline Depresión & 152 & 156 & 129 & 111 & 76 & 624 \\
\hline Trastorno bipolar & 17 & 12 & 14 & 39 & 17 & 99 \\
\hline Total & 685 & 672 & 595 & 587 & 422 & 2961 \\
\hline
\end{tabular}

Nota. Fuente: REDALYC y PSICODOC.

Figura 2. Número total de artículos publicados en Iberoamérica entre los años 2003 a 2007, en áreas del campo psicopatología.

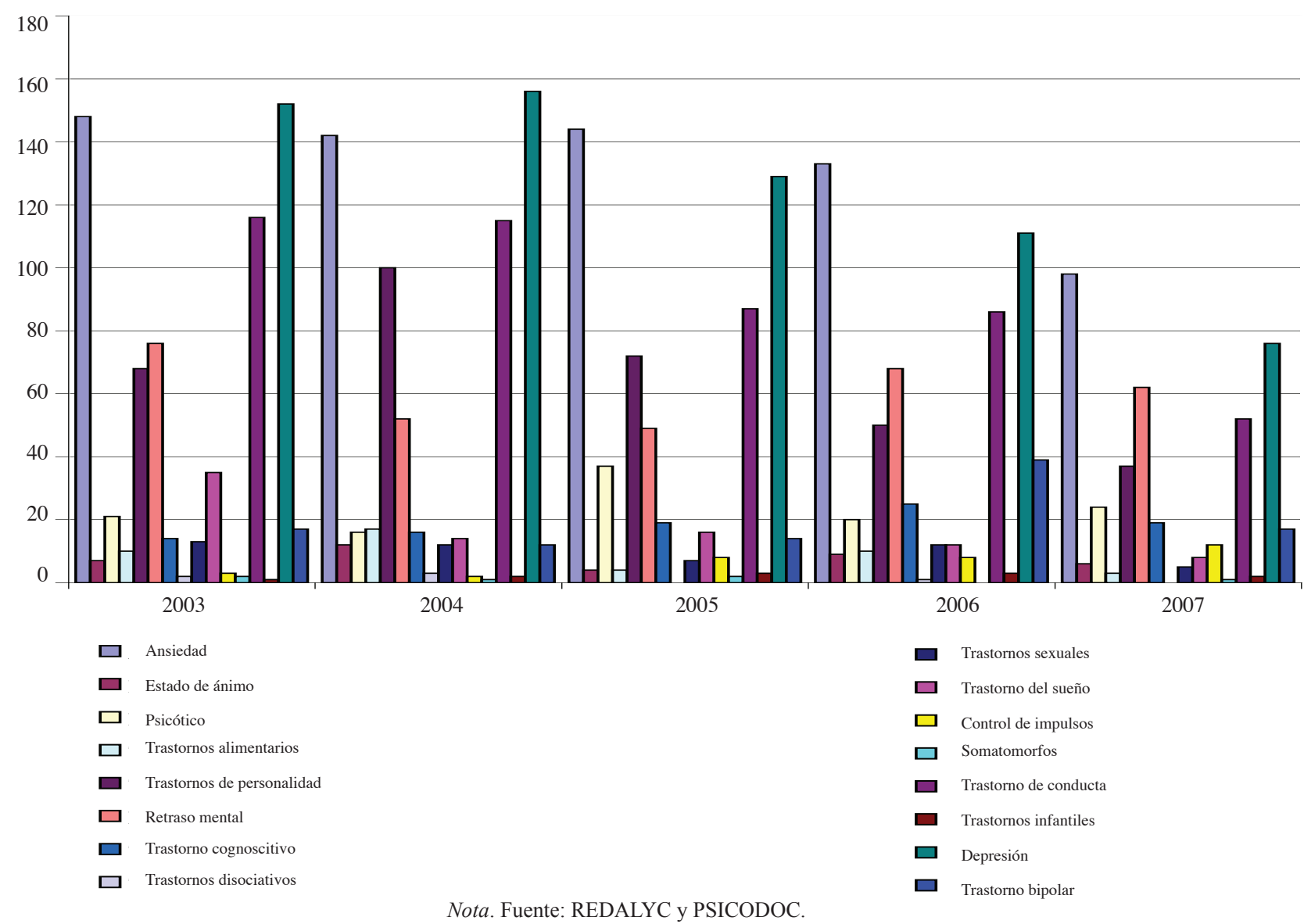


realizada en REDALYC y PSICODOC con dichas palabras, de los cuales 27 fueron publicados por España y seis por Colombia, siendo los países con mayor coincidencia en la búsqueda.

Trastornos de personalidad. Con las palabras "Trastornos de personalidad" en REDALYC se encontraron 13 artículos, 11 artículos de revistas de España, uno de Argentina y uno de Chile. Asimismo en PSICODOC se encontró 314 artículos de revistas, 85 de España, 15 de México y ocho de Colombia. Entre las dos bases de datos, la búsqueda con estas palabras arrojó 327 artículos, de los cuales 96 eran de revistas de España.

Retraso mental. En REDALYC se encontró tres artículos de revistas, uno de España, uno de Argentina y uno de Colombia. Con la misma palabra clave se encontró en PSICODOC 304 artículos, destacándose España con 93 artículos, Brasil con 41 artículos y México con 23. Con las palabras "Retraso mental" se halló un total de 307 artículos de revistas en REDALYC y PSICODOC, estando España a la cabeza con 94 artículos y Brasil con 41 artículos.

Trastorno cognoscitivo. Con "Trastorno cognoscitivo" como palabras claves, se obtuvo como resultado en la búsqueda de REDALYC un artículo de una revista española. En PSICODOC se encontró un total de 92 artículos, 57 de los cuales eran de revistas españolas, 16 de Chile y nueve de Argentina. En total se hallaron 93 artículos, siendo España el país de origen con mayor coincidencia en la búsqueda.

Trastornos disociativos. Con estas palabras claves, en la búsqueda de REDALYC se encontró un artículo publicado por México. De igual forma en PSICODOC se encontró cinco artículos, dos de España, dos de México y uno de Brasil, para un total de seis artículos.

Trastornos sexuales. En REDALYC se encontró un artículo de una revista española, publicado en el año 2003. Asimismo en PSICODOC se halló 48 artículos con las mismas palabras, 32 de España, ocho de México y cinco de Argentina.

Trastorno de sueño. En el primer sistema de información con las palabras "Trastorno del sueño" se encontró un artículo publicado en el 2005 en una revista colombiana. En PSICODOC, por su parte, se halló 84 artículos, 43 de revistas españolas, para un total de 85 artículos.

Somatomorfos. Con esta palabra clave no se encontraron coincidencias en REDALY. En PSICODOC se encontró seis artículos, tres de España, dos de Colombia y uno de Chile.

Control de impulsos. Con las palabras "Control de impulsos" se encontró en REDALYC cuatro artículos de revista y en PSICODOC 29 artículos, para un total de 33 artículos, siendo España el que reporta mayor número de publicaciones con 25 artículos de revista, seguido por México con ocho artículos de revistas.
Trastorno de conducta. Con estas palabras claves se encontró en REDALYC un artículo de una revista española publicado en el 2003, mientras que en PSICODOC se halló 455 artículos. España fue el país con el mayor número de coincidencias con 320 artículos, segundo Brasil con 42 artículos y tercero Colombia con 31 artículos de revista.

Trastornos infantiles: Con estas palabras no se encontró ninguna coincidencia en la búsqueda que se realizó REDALYC. En el sistema de información PSICODOC con la misma palabra clave se encontró 11 registros de los cuales ocho eran de revistas españolas, dos de revistas mexicanas $\mathrm{y}$ uno de una revista colombiana.

Depresión. En REDALYC se encontró 56 artículos de revista, siendo España el país con mayor producción con 36 artículos, seguido por Colombia con 12 artículos y México y Chile con cuatro artículos cada uno. En PSICODOC se encontró con la misma palabra clave 568 artículos de revista. Los países que reportaron mayor coincidencia en la búsqueda fueron España con 367 artículos de revista, Brasil con 42 artículos y Colombia con 38 artículos. En total se registraron 624 artículos.

Trastorno bipolar. En REDALYC se halló cuatro artículos, tres de revistas colombianas y uno de España. En PSICODOC se encontró 95 artículos de revistas, siendo España el país con mayor publicación con 53 trabajos.

\section{Evaluación psicológica}

Con estas palabras claves en la búsqueda en REDALYC, se halló 14 artículos de revistas, de los cuales nueve eran de revistas de España, tres de Argentina y dos de Colombia. En PSICODOC se encontró un total de 759 artículos de revistas, siendo España el país con mayor número de artículos al contar 632. En total se contaron 773 artículos (véase la Tabla 1 y la Figura 1).

\section{Diagnóstico psicológico}

La búsqueda en REDALYC no arrojó ninguna coincidencia; sin embargo, en PSICODOC dicha búsqueda dio como resultado 12 artículos, de los cuales 10 eran de España y dos de Argentina (véase la Tabla 1 y la Figura 1).

\section{Formación del psicólogo}

Con estas palabras claves en REDALYC no se encontró ninguna coincidencia. En cambio en PSICODOC con la misma palabra clave se halló 15 artículos, encontrándose España en el primer lugar con 11 artículos, Brasil con dos artículos y Colombia y Argentina cada uno con un artículo (véase la Tabla 1 y la Figura 1).

\section{Enfoque terapéutico}

Con las palabras claves "Enfoque terapéutico" se encontró un artículo en REDALYC y 153 en PSICODOC, para 
un total de 154 artículos, siendo el 2006 el año de mayor producción con 55 artículos (véase la Tabla 1 y la Figura 1). A continuación se exponen los resultados obtenidos con las palabras claves específicas de este campo (véase la Tabla 3 y la Figura 3).

Terapia cognitiva. En la búsqueda hecha en REDALYC con las palabras "Terapia cognitiva" se encontró un total de 12 artículos de revista, de los cuales nueve eran de revistas de España y tres de Colombia. En cambio en PSICODOC se encontró con la misma palabra clave un total de 348 artículos de revistas, de los cuales 123 eran de España, 45 de México y 25 de Colombia. Por consiguiente, en REDALYC y PSICODOC el total de artículos encontrados con la palabra clave "Terapia cognitiva" fue de 360 artículos, siendo España el país de origen de las revistas con mayor producción, con 132 artículos.

Terapia de conducta. Con "Terapia de conducta" en la búsqueda en REDALYC se registraron seis artículos publicados por revistas españolas. En PSICODOC se encontró 323 artículos, en donde los países con mayor coincidencia en la búsqueda fueron España con 197 artículos, Brasil con 43 artículos y Colombia con 19 artículos. El total de artículos fue de 329.

Terapia cognitivo conductual. Con las palabras claves "Terapia cognitivo conductual" no se obtuvo ninguna coincidencia en el sistema de información REDALYC. Por otra parte, en PSICODOC se encontró 38 artículos, 16 publicados por revistas españolas.

Tabla 3. Número total de artículos publicados en Iberoamérica entre los años 2003 a 2007, en áreas del campo enfoque terapéutico.

\begin{tabular}{lcccccc}
\hline Área & 2003 & 2004 & 2005 & 2006 & 2007 & Total \\
\hline Terapia cognitiva & 100 & 80 & 64 & 76 & 40 & 360 \\
Terapia de conducta & 83 & 69 & 47 & 106 & 24 & 329 \\
Terapia cognitivo conductual & 3 & 6 & 13 & 16 & - & - \\
Terapia humanista & 1 & - & 1 & 1 & - & 2 \\
Psicodinámico & 1 & 1 & 8 & 8 & 9 & 4 \\
Terapia sistémica & 6 & 15 & 134 & 206 & 75 & 780 \\
Total & 194 & 171 & & & \\
\hline
\end{tabular}

Nota: Ausencia de registros. Fuente: REDALYC y PSICODOC.

Figura 3. Número total de artículos publicados en Iberoamérica entre los años 2003 a 2007, en áreas del campo enfoque terapéutico.

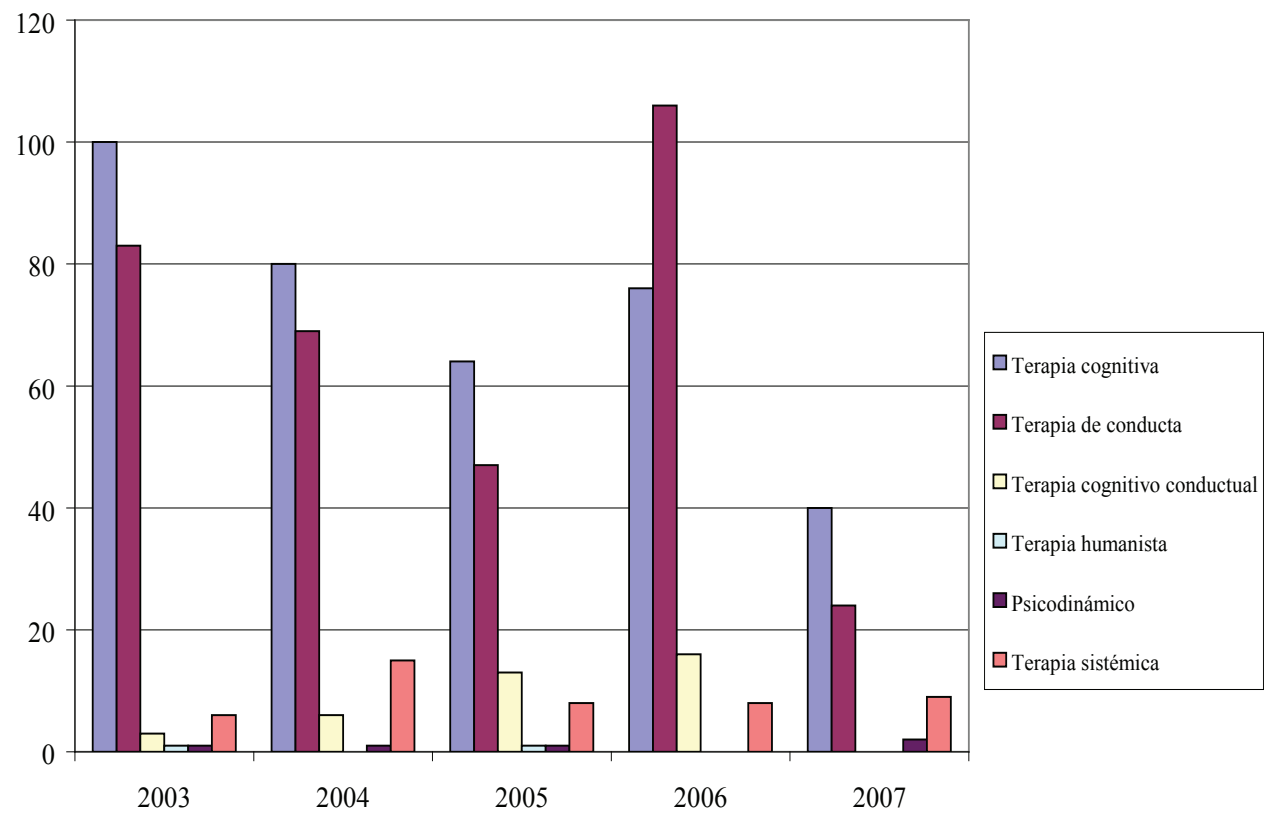

Nota. Fuente: REDALYC y PSICODOC. 
Terapia humanista. La búsqueda en REDALYC no mostró coincidencias, mientras que en PSICODOC se encontraron dos artículos publicados en España.

Psicodinámica. No se encontraron resultados en REDALYC, aunque en PSICODOC se hallaron cinco artículos, de los cuales cuatro fueron publicados en España y uno en Argentina.

Terapia sistémica. En REDALYC se encontraron dos artículos publicados en Colombia y en PSICODOC se registraron 42 artículos de revistas, de los cuales 19 eran de España, nueve de Colombia y siete de Portugal.

\section{Psicoterapia}

Con la palabra clave "Psicoterapia" en la búsqueda de REDALYC se encontraron 87 artículos de revista, 24 de España, 23 de Colombia y 14 de Brasil. En PSICODOC se halló 888 artículos, siendo España el país con mayor producción con 563 artículos de revista, seguido por Colombia con 63 y Argentina con 53. En total se registraron 975 artículos, resaltando España como el país con mayor número de artículos con 587 y el año 2004 como el de mayor producción.

\section{Número de artículos por el país de origen de las revistas}

Como se puede observar en la Tabla 4, el país de origen de las revistas con mayor número de artículos fue España con 3323 trabajos, seguido por Colombia con 343 artículos, México con 218 artículos, Brasil con 277 y Argentina con 130 publicaciones. Los demás países registrados tuvieron una publicación inferior a 50 artículos.

Tabla 4. Número de artículos por el país de origen de las revistas.

\begin{tabular}{cc}
\hline País & Total \\
\hline España & 3323 \\
Colombia & 343 \\
México & 218 \\
Brasil & 277 \\
Argentina & 130 \\
Portugal, Uruguay, Venezuela, Chile, Costa Rica, Cuba, Perú, El Salvador & Menos de 50 artículos \\
\hline Nota. Fuente: REDALYC y PSICODOC.
\end{tabular}

\section{Discusión}

El objetivo de este trabajo fue realizar un estudio exploratorio de carácter bibliométrico, sobre las tendencias de los artículos científicos en psicología clínica en Iberoamerica, con base en el número de artículos que se pueden recuperar de los sistemas de información REDALYC y PSICODOC, en los campos psicopatología, evaluación psicológica, diagnóstico psicológico, formación del psicólogo, enfoque terapéutico y psicoterapia, realizándose adicionalmente un análisis más específico a nivel de las áreas psicopatología y enfoque terapéutico. En resumen, se encontró que las áreas con mayor número de coincidencias fueron "Psicoterapia" con 975 artículos de revista, "Evaluación psicológica" con 773 artículos, "Psicopatología” con 769 artículos y "Enfoque terapéutico" con 154 artículos, siendo baja la productividad en los campos de "Formación del psicólogo" (15 artículos) y "Diagnóstico psicológico" (12 artículos).

Para el caso específico del campo de psicopatología, las áreas “Ansiedad”, "Depresión”, "Trastorno de conducta”, "Trastornos de la personalidad" y "Retraso mental", constituyeron los ejes temáticos de mayor producción con más de 300 registros. En cambio, se observó una baja producción en los temas de "Trastornos disociativos", "Somatomorfos",
"Trastornos infantiles", Estado de ánimo", "Trastornos alimentarios", "Trastornos sexuales" y "Control de impulsos", con un número de registros que oscilaba entre 6 y 49. Las restantes áreas ("Trastorno cognoscitivo", "Psicótico", "Trastorno del sueño" y "Trastorno bipolar") evidenciaron una productividad "media", con registros cuyo número oscilaba entre 85 y 118.

Por otra parte, los enfoques psicológicos que presentaron el mayor número de coincidencias fueron "Terapia cognitiva" con 360 artículos de revista y "Terapia de conducta" con 329 artículos de revista, seguidos por la "Terapia sistémica" con 46 artículos y "Terapia cognitivo conductual" con 38 registros, mientras que las palabras claves "Psicodinámico" y "Terapia humanista" arrojaron un bajo número de coincidencias (cinco y dos, respectivamente).

Estos datos señalan que en la región existe un marcado interés académico por la psicoterapia, la evaluación psicológica y la psicopatología y una menor atención por el enfoque terapéutico, mientras que la formación del psicólogo y el diagnóstico psicológico parecen temas de poco interés en la misma. Estos datos revelan, asimismo, que la investigación en Iberoamérica sigue mostrando una preponderancia por temas habituales de la psicología clínica (excepto el diagnóstico psicológico), mientras que otros 
menos "tradicionales" como la formación del psicólogo continúan siendo poco explorados, a pesar de su relevancia por aspectos como la preparación académica de pregrado y postgrado, los principios y estándares éticos y la legislación que en cada país reglamenta el ejercicio de la profesión. En Colombia, por ejemplo, el tema de la formación del psicólogo ha despertado una gran atención en los círculos académicos y profesionales, por la aprobación de leyes tales como la Ley 1090 del 6 de septiembre de 2006 (República de Colombia, 2006), en la cual se establecen los requisitos mínimos para ejercer la profesión y se regula su ejercicio en los campos aplicados, en la docencia y en la investigación y la Ley 1164 del 3 de octubre de 2007 (República de Colombia, 2007), la cual reglamenta la formación que deben tener los profesionales de la salud. Por lo tanto, en éste y otros países de la región debería haber un mayor interés académico sobre este tema, que se evidencie en un mayor número de artículos sobre el mismo.

Por otra parte y aunque el diagnóstico psicológico es una de las actividades más comunes del psicólogo en el campo clínico, no parece existir mucho interés académico con respecto al mismo, en comparación con el más amplio campo de la evaluación psicológica. Si bien ello es coherente con la necesidad de que el papel del profesional no se limite al diagnóstico, sino a la evaluación comprehensiva del caso, de manera que ello lleve a un mejor proceso de atención en donde las estrategias y el plan de intervención respondan a las necesidades del usuario (Bellack \& Hersen, 1993; Fernández-Ballesteros, 2004), algunos aspectos claman por una mayor atención con respecto a este tema en la región, como las categorías diagnósticas que se han planteado recientemente, las propuestas de otros sistemas diagnósticos, la comunicación del diagnóstico con los consultantes y con otros profesionales, entre otros.

En el campo de la psicopatología, por otra parte, es evidente que existe un gran interés por los temas de la ansiedad y la depresión, que indica que en la región existe un significativo número de grupos de investigación que trabajan alrededor del diagnóstico, la evaluación y el tratamiento de los trastornos de ansiedad y el estado de ánimo. No obstante, es posible que este resultado refleje los intereses de enfoques como la terapia cognitiva y la terapia de conducta, los cuales han trabajado tradicionalmente alrededor de estos temas, como lo muestra el alto número de tratamientos bien establecidos o probablemente eficaces para los trastornos de ansiedad y del estado del ánimo realizados desde estos enfoques (véase Pérez \& Fernández, 2001). Por otra parte, la prevalencia de este tipo de trastornos parece bastante elevada en los países de la región, como se desprende de algunos estudios como el realizado en Chile con 2978 individuos (Vicente, Rioseco, Saldivia, Kohn \& Torres, 2002), en donde se encontró una prevalencia vida de $16.2 \%$ de trastornos por ansiedad y de $15 \%$ para los trastornos afectivos. En Colombia el estudio nacional de salud mental (Posada-Villa, Aguilar-Gaxiola, Magaña \& Gómez, 2004) arrojó resultados similares, con una prevalencia vida de $19.3 \%$ para los trastornos de ansiedad y de $15 \%$ para los trastornos afectivos.

En comparación con estos temas tradicionales, resulta bastante aliciente que haya un interés similar por otro que ha llamado tradicionalmente menos la atención: el trastorno de conducta, cuya prevalencia alcanza entre el 6 y el $16 \%$ de los varones menores de dieciocho años de edad y entre el 2 y el $9 \%$ de las mujeres de la misma edad (APA, 2002). Sin embargo, es posible que este interés también refleje una inclinación de los investigadores de la terapia de conducta, desde cuyo enfoque se han desarrollado y publicado diferentes técnicas y programas de tratamiento para los problemas de conducta infantil (véase Martin \& Pear, 1999). Algo similar pasaría con el retraso mental, para el cual se han desarrollado varias alternativas de rehabilitación de corte conductual desde los años '70s (véase Ribes, 1974). Otro tema que llama la atención por su elevado número de registros es el de los trastornos de personalidad, que señala que en la región existe una gran atención por su tema en los círculos académicos. En contraposición, temas como los trastornos disociativos y somatomorfos, los trastornos infantiles, el estado de ánimo, los trastornos alimentarios, los trastornos sexuales y el control de impulsos, parecen de menor interés comparados con los anteriores, a pesar de que sus síntomas clínicos son similarmente serios o incluso más graves. Los trastornos cognoscitivos, los trastornos psicóticos, los trastornos del sueño y el trastorno bipolar son temas que parecen atraer medianamente la atención académica en la región.

En resumen, los datos obtenidos sobre las áreas específicas del campo "Psicopatología" señalan que en la región existe toda una gama de temas que no han sido abordados suficientemente a nivel académico y que los esfuerzos investigativos deberían dirigirse al estudio del diagnóstico, la evaluación, el tratamiento y la prevención de trastornos distintos a los de ansiedad y el estado del ánimo, cuya investigación ameritaría estudios meta-analíticos y trabajos de revisión para racionalizar su abordaje en el futuro.

En cuanto al enfoque terapéutico, es evidente un mayor interés por la terapia cognitiva y la terapia conductual, mientras que éste parece mucho más bajo o casi nulo en relación con las demás perspectivas (sistémico, humanista y psicodinámico). Es posible que ello se deba a que estos dos tipos de terapia suelen estar enmarcados dentro de un enfoque más general llamado "terapia y modificación del comportamiento", en el cual existe una fuerte tradición por la investigación y la publicación de los resultados de los estudios dirigidos a validar sus planteamientos teóricos y la efectividad de sus tratamientos psicológicos (Labrador, Cruzado \& Muñoz, 1998). El alto número de artículos sobre terapia cognitiva, por otra parte, muestra un elevado interés académico por el tema que ya había sido observado (con un 
período de tiempo más largo), en un estudio bibliométrico sobre las tesis de pregrado y postgrado sobre terapia y modificación del comportamiento realizadas en la ciudad de Bogotá, Colombia (Rey \& Acevedo, 2005). En este estudio, efectivamente, se encontró que el número de tesis sobre técnicas o programas de tratamiento cognitivos era nulo en los años '70s, elevándose en los años ' 80 s, en los '90s y los años 2000 a 2003. La terapia conductual, por otra parte, parece gozar del mismo interés que comenzó a evidenciarse en la región a principios de los años '70s (véase Ardila, 1974), aunque los datos no son coherentes con los del ya citado estudio de Rey y Acevedo (2005), en el que se encontró que el interés por las técnicas de tratamiento basados en el condicionamiento operante disminuyó ostensiblemente en los años ' $90 \mathrm{~s}$ s, al pasar de 21 tesis en los '70s y 20 en los ' 80 s, a sólo 6 en los '90s y una entre los años 2000 y 2003.

De otro lado, resulta muy curioso que se haya encontrado un número relativamente bajo de artículos sobre terapia cognitivo conductual, ya que los tratamientos psicológicos diseñados desde este enfoque terapéutico gozan de un gran respaldo empírico para muchos trastornos mentales (McGinn \& Sanderson, 2001). Así, por ejemplo, existen tratamientos cognitivo conductuales bien establecidos para la bulimia, la ansiedad generalizada, la fobia simple, la agorafobia y el trastorno bipolar y probablemente eficaces para la hipocondría y la dependencia a la cocaína y la heroína (Pérez \& Fernández, 2001). Dado que el desarrollo de este tipo de tratamientos es relativamente reciente, es de esperar un aumento en el número de artículos sobre el mismo en la región, en la medida que estos tratamientos sean adaptados para las poblaciones locales o se desarrollen nuevas propuestas terapéuticas desde dicho enfoque.

El número de artículos por país de origen de las revistas muestra, finalmente, una gran preponderancia de las revistas españolas sobre las de otros países iberoamericanos, que se puede explicar no solamente porque en este país existe un gran respaldo financiero para la investigación, sino por el alto número de revistas científicas en psicología que se publican en el mismo, indexadas o incorporadas en sistemas de información científica. Motivos similares explicarían en menor proporción el número de artículos publicados en revistas de Colombia, México, Brasil y Argentina, ya que en estos países se observa un número importante de revistas científicas en psicología y disciplinas afines, en comparación con los demás de la región.

Dada la naturaleza de este análisis, se pueden enumerar algunas limitaciones que deberían ser subsanadas en futuras investigaciones sobre este tema. Por un lado, no se utilizaron palabras claves en otros idiomas distintos al castellano, por lo que no fue posible examinar de manera válida el nivel de producción de países como Brasil y Portugal. Ya que no se incluyó en el análisis revistas en inglés y, por consiguiente, vinculadas a sistemas de información como PSYCINFO e ISI WEB OF KNOWLEDGE, es posible que no se hayan considerado muchos artículos que podrían reflejar intereses distintos por parte de los académicos en la región. Por otra parte, es posible que otras palabras claves no utilizadas en este análisis, revelen otros datos de interés en relación con el tema y otras tendencias igual de importantes a las encontradas en el mismo.

\section{Referencias}

Agudelo, D., Bretón-López, J. \& Buela-Casal, G. (2003). Análisis bibliométrico de las revistas de Psicología Clínica editadas en castellano. Psicothema, 15, 507-516.

Ardila, R. (1974). Prólogo. En R. Ardila (Comp.), El análisis experimental del comportamiento: La contribución latinoamericana (pp. 9-12). México: Trillas.

Asociación Psiquiátrica Americana (2002). Manual Diagnóstico y Estadístico de los Trastornos Mentales, Cuarta Edición, Texto Revisado. Madrid: Masson.

Barlow, D. \& Durand, V. M. (2001). Psicología anormal: Un enfoque integral. México: Thomson.

Bellack, A. S. \& Hersen, M. (Eds) (1993). Manual práctico de evaluación de conducta. Bilbao: Desclée de Brouwer.

Chambless, D .L. \& Hollon, S. D. (1998). Defining empirically supported therapies. Journal of Consulting and Clinical Psychology, 66, 7-18.

Fernández-Ballesteros, R. (2004). Introducción a la evaluación psicológica II. Madrid: Pirámide.

Halgin, R. \& Whitbourne, S. (2003). Psicología de la anormalidad: Perspectivas clínicas sobre desórdenes psicológicos (4 Ed.). México: McGraw Hill.

Labrador, F. J., Cruzado, J. A. \& Muñoz, M. (1998). Manual de técnicas de modificación y terapia de conducta. Madrid: Pirámide.

LATINDEX (2008, noviembre 17). LATINDEX: Sistema Regional de Información en Línea para Revistas Cientificas de América Latina, el Caribe, España y Portugal. Recuperado en noviembre 17, 2008, disponible en http://www.latindex.unam.mx/index.html?opcion=1\&T=G\&nivel tema $=5 \&$ renglon $=6 \& \mathrm{X}=$

Londoño, C. \& Valencia, S. C. (2005). La investigación en el proceso de soporte empírico de las terapias: Alcances y limitaciones. Acta Colombiana de Psicología, 13, 163-181.

Llobell, J. P., Frías, M. D. \& Monterde, H. (2004). Tratamientos psicológicos con apoyo empírico y práctica clínica basada en la evidencia. Papeles del Psicólogo, 87, 1-8.

Martin, G. \& Pear, J. (1999). Modificación de conducta, qué es y cómo aplicarla. Madrid: Prentice Hall.

McGinn, L. K. \& Sanderson, W. C. (2001). What allows cognitive behavioral therapy to be brief: Overview, efficacy, and crucial factors facilitating brief treatment. Clinical Psychology: Science and Practice, 8, 23-37.

Nietzel, M. T., Bernstein, D. A. \& Milich, R. (1998). Introduction to clinical psychology ( $5^{\mathrm{a}} \mathrm{Ed}$.). New Jersey: Prentice Hall.

Nietzel, M. T., Bernstein, D. A. \& Milich, R. (1998). Introduction to clinical psychology ( $5^{\mathrm{a}}$ Ed.). New Jersey: Prentice Hall.

Peña, T. E. (2005). Psicología y psiquiatría: Un análisis de sus identidades. Cuadernos de Psicología, 1, 11-32.

Pérez, M. \& Fernández, J. R. (2001). El grano y la criba de los tratamientos psicológicos. Psichotema. 13, 523-529.

PSICODOC (2008, noviembre 17). PSICODOC: Base de datos bibliográfica de psicología. Recuperado en noviembre 17, 2008, disponible en http://psicodoc.copmadrid.org/psicodoc.htm.

Posada-Villa, J. A., Aguilar-Gaxiola, S. A., Magaña, C. G. \& Gómez, L. C. (2004). Prevalencia de trastornos mentales y uso de servicios: Resultados preliminares del Estudio nacional de salud mental. Colombia, 2003. Revista Colombiana de Psiquiatría, 33, 241-262.

REDALYC (2008, noviembre 17). Red de Revistas Cientificas de América Latina y el Caribe, España y Portugal: Presentación. Recuperado en noviembre 17, 2008, disponible en http://redalyc.uaemex.mx/src/ auxHemeroteca/acerca.html. 
República de Colombia (2006). Ley 1090 del 6 de septiembre de 2006 "Por la cual se reglamenta el ejercicio de la profesión de psicología, se dicta el código deontológico y bioético y otras disposiciones”. Bogotá: Congreso de la República.

República de Colombia (2007). Ley 1164 del 3 de octubre de 2007 "Por la cual se dictan disposiciones en materia del talento humano en salud”. Bogotá: Congreso de la República.

Resnick, J. H. (1991). Finally, a definition of clinical psychology: A message from the President, Division 12. The Clinical Psychologist 44, 3-11.

Rey, C. A. \& Acevedo, A. (2005). Análisis bibliométrico de las tesis de pregrado y postgrado realizadas en Bogotá, sobre la implementación de programas de Terapia y Modificación del Comportamiento. Acta Colombiana de Psicología, 14, 97-111.
Ribes, E. (1974). Técnicas de modificación de conducta: Su aplicación al retardo en el desarrollo. México: Trillas.

Sarason, I. G. \& Sarason, B. R. (1996). Psicología anormal: El problema de la conducta inadaptada ( $7^{\mathrm{a}}$ Ed.). México: Prentice Hall.

Sue, D., Sue, D. \& Sue, S. (1996). Comportamiento anormal (4 $4^{\mathrm{a}} \mathrm{Ed}$.). México: McGraw Hill.

Trull, E. J. \& Phares, T. J. (2003). Psicología clínica: Conceptos, métodos y aspectos prácticos de la profesión ( $6^{\mathrm{a}} \mathrm{Ed}$.). México: Thomson.

Vera-Villarroel, P. \& Lillo, S. (2006). La investigación actual en psicología clínica en Chile: Un análisis a partir de la producción. Terapia Psicológica, 24, 221-230.

Vicente, B., Rioseco, P., Saldivia, S., Kohn, R. \& Torres, S. (2002). Estudio chileno de prevalencia de patología psiquiátrica (DSM-III-R/CIDI) (ECPP). Revista Médica de Chile, 130, 527-536. 